\title{
Pure laparoscopic radical resection for type IIIa hilar cholangiocarcinoma
}

\author{
Cheng-wu Zhang ${ }^{1} \cdot$ Jie Liu ${ }^{1}$ - De-fei Hong ${ }^{1} \cdot$ Zhi-fei Wang $^{1} \cdot$ Zhi-ming Hu$^{1}$ • \\ Dong-shen Huang ${ }^{1} \cdot$ Min-jie Shang ${ }^{1} \cdot$ Wei-feng Yao ${ }^{1}$
}

Received: 21 March 2017/Accepted: 14 July 2017 / Published online: 4 August 2017

(c) The Author(s) 2017. This article is an open access publication

\begin{abstract}
Background Pure laparoscopic radical resection of hilar cholangiocarcinoma is still a challenging procedure, in which laparoscopic lymphadenectomy, hemihepatectomy with caudate lobectomy, and hepaticojejunostomy were included [1-4]. Relative report is rare in the world up to now. Hilar cholangiocarcinoma has a poor prognosis, especially when it occurs with lymph node metastasis or vessel invasion $[5,6]$. We recently had a patient who underwent a pure laparoscopic extended right hepatectomy and lymph node dissection and hepaticojejunostomy for a type IIIa hilar cholangiocarcinoma.

Methods The tumor was $20 \times 15 \times 12 \mathrm{~mm}$ in diameter and located in the right bile duct and common hepatic duct. Radiological examination showed that hepatic artery and portal vein was not invaded. After the division and mutilation of the right hepatic artery and the right portal vein, short hepatic veins were divided and cut off with clip and ultrasound knife from the anterior face of the vena cava. Mobilization was performed after the devascularization of the right liver, followed by the transection of liver parenchymal with CUSA and ultrasound knife. Finally, left hepatic bile duct jejunum Roux-en-Y reconstruction was performed.
\end{abstract}

Electronic supplementary material The online version of this article (doi:10.1007/s00464-017-5741-4) contains supplementary material, which is available to authorized users.

Jie Liu

atianlx1226@163.com

1 Department of Hepatopancreatobiliary Surgery and Minimally, Invasive Surgery, Zhejiang Provincial People's Hospital, Hangzhou 310014, Zhejiang, China
Results This patient underwent successfully with a totally laparoscopic procedure. An extended right hepatectomy (right hemihepatectomy combined with caudate lobectomy) and complete lymph node dissection and hepaticojejunostomy were performed in this operation. The operation time was nearly $590 \mathrm{~min}$, and the intraoperative blood loss was about $300 \mathrm{ml}$. No obvious complication was observed and the postoperative hospital stay was 11 days. The final diagnosis of the hilar cholangiocarcinoma with no lymph node metastasis was pT2bN0M0 stage II (American Joint Committee on Cancer, AJCC).

Conclusions Pure laparoscopic resection for hilar cholangiocarcinoma was proved safe and feasible, which enabled the patient to recover early and have an opportunity to receive chemotherapy as soon as possible. We present a video of the described procedure.

Keywords Laparoscopic resection · Hilar cholangiocarcinoma $\cdot$ Surgical technique

\section{Compliance with ethical standards}

Disclosures Cheng-wu Zhang, Jie Liu, De-fei Hong, Zhi-fei Wang, Zhi-ming Hu, Dong-shen Huang, Min-jie Shang, and Wei-feng Yao have no conflict of interest or financial ties to disclose.

Open Access This article is distributed under the terms of the Creative Commons Attribution 4.0 International License (http://creativecommons. org/licenses/by/4.0/), which permits unrestricted use, distribution, and reproduction in any medium, provided you give appropriate credit to the original author(s) and the source, provide a link to the Creative Commons license, and indicate if changes were made.

\section{References}

1. Puntambekar S, Sharma V, Kumar S, Mitkare S, Joshi G, Parikh H (2016) Laparoscopic management of hilar cholangiocarcinoma: a case report. Indian J Surg 78:57-59 
2. Lee W, Han HS, Yoon YS, Cho JY, Choi Y, Shin HK, Jang JY, Choi H (2015) Laparoscopic resection of hilar cholangiocarcinoma. Ann Surg Treat Res 89:228-232

3. Cho A, Yamamoto H, Kainuma O, Muto Y, Yanagibashi $\mathrm{H}$, Tonooka T, Masuda T (2014) Laparoscopy in the management of hilar cholangiocarcinoma. World J Gastroenterol 20:15153-15157

4. Machado MA, Makdissi FF, Surjan RC, Mochizuki M (2012) Laparoscopic resection of hilar cholangiocarcinoma. J Laparoendosc Adv Surg Tech A 22:954-956
5. Kang MJ, Jang JY, Chang J, Shin YC, Lee D, Kim HB, Kim SW (2016) Actual long-term survival outcome of 403 consecutive patients with hilar cholangiocarcinoma. World J Surg 40:2451-2459

6. Buettner S, Margonis GA, Kim Y, Gani F, Ethun CG, Poultsides G, Tran T, Idrees K, Isom CA, Fields RC, Krasnick B, Weber SM, Salem A, Martin RC, Scoggins CR, Shen P, Mogal HD, Schmidt C, Beal E, Hatzaras I, Shenoy R, Maithel SK, Pawlik TM (2016) Conditional probability of long-term survival after resection of hilar cholangiocarcinoma. HPB (Oxford) 18:510-517 